\title{
The CIELO collaboration: Progress in international evaluations of neutron reactions on Oxygen, Iron, Uranium and Plutonium
}

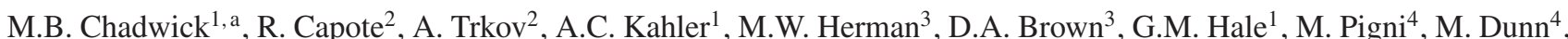
L. Leal ${ }^{5}$, A. Plompen ${ }^{6}$, P. Schillebeeck ${ }^{6}$, F.-J. Hambsch ${ }^{6}$, T. Kawano ${ }^{1}$, P. Talou ${ }^{1}$, M. Jandel ${ }^{1}$, S. Mosby ${ }^{1}$, J. Lestone ${ }^{1}$, D. Neudecker ${ }^{1}$, M. Rising ${ }^{1}$, M. Paris ${ }^{1}$, G.P.A. Nobre ${ }^{3}$, R. Arcilla ${ }^{3}$, S. Kopecky ${ }^{6}$, G. Giorginis ${ }^{6}$, O. Cabellos ${ }^{7}$, I. Hill ${ }^{7}$, E. Dupont ${ }^{7}$, Y. Danon ${ }^{8}$, Q. Jing 9 , G. Zhigang ${ }^{9}$, L. Tingjin 9 , L. Hanlin ${ }^{10}$, R. Xichao ${ }^{10}$, W. Haicheng ${ }^{10}$, M. Sin ${ }^{11}$, E. Bauge ${ }^{12}$, P. Romain ${ }^{12}$, B. Morillon ${ }^{12}$, G. Noguere ${ }^{13}$, R. Jacqmin ${ }^{13}$, O. Bouland ${ }^{13}$, C. De Saint Jean ${ }^{13}$, V.G. Pronyaev ${ }^{14}$, A. Ignatyuk ${ }^{14}$, K. Yokoyama ${ }^{15}$, M. Ishikawa ${ }^{15}$, T. Fukahori ${ }^{15}$, N. Iwamoto ${ }^{15}$, O. Iwamoto $^{15}$, S. Kuneada ${ }^{15}$, C.R. Lubitz ${ }^{16}$, G. Palmiotti ${ }^{17}$, M. Salvatores ${ }^{17}$, I. Kodeli ${ }^{18}$, B. Kiedrowski ${ }^{19}$, D. Roubtsov ${ }^{20}$, I. Thompson ${ }^{21}$, S. Quaglioni ${ }^{21}$, H.I. Kim ${ }^{22}$, Y.O. Lee ${ }^{22}$, A.J. Koning ${ }^{2}$, A. Carlson ${ }^{23}$, U. Fischer ${ }^{24}$, and I. Sirakov ${ }^{25}$

1 Los Alamos National Laboratory, Los Alamos, NM87545, USA

2 NAPC-Nuclear Data Section, International Atomic Energy Agency, Vienna, Austria

3 Brookhaven National Laboratory, Brookhaven, USA

${ }^{4}$ Oak Ridge National Laboratory, Oak Ridge, TN 37831, USA

5 Institut de Radioprotection et de Sureté Nucleaire, Paris, France

${ }^{6}$ European Commission, Joint Research Center, Retieseweg 111, 2440 Geel, Belgium

7 Nuclear Energy Agency, OECD, Paris

${ }^{8}$ Rensselaer Polytechnic Institute, Troy, NY, USA

9 China Nuclear Data Center, PO Box 275-41, Beijing 102413, P.R. China

10 China Institute of Atomic Energy, PO Box 275-41, Beijing 102413, P.R. China

11 Nuclear Physics Department, Bucharest University, Bucharest-Magurele, Romania

12 CEA, DAM Ile de France, 91297 Arpajon, France

13 CEA, Nuclear Energy Division, Cadarache, Saint-Paul-lez-Durance, France

${ }^{14}$ Institute of Physics and Power Engineering, Obninsk, Russia

15 Japan Atomic Energy Agency, Tokai-mura, Japan

16 Knolls Atomic Power Laboratory, Schenectady, NY, USA

17 Idaho National Laboratory, Idaho Falls, ID, USA

18 Jozef Stefan Institute, Ljubljana, Slovenia

19 Department of Nuclear Engineering, Michigan University, Michigan, USA

${ }^{20}$ Chalk River Laboratories, AECL, Chalk River, Ontario, Canada

21 Lawrence Livermore National Laboratory, Livermore, CA, USA

22 Korean Atomic Energy Research Institute, Daejeon, South Korea

23 National Institute of Standards and Technology, Gaithersburg, MD, USA

${ }^{24}$ Karlsruhe Institute of Technology, Karlsruhe, Germany

25 Institute for Nuclear Research and Nuclear Energy, Sofia 1784, Bulgaria

\begin{abstract}
The CIELO collaboration has studied neutron cross sections on nuclides that significantly impact criticality in nuclear technologies $-{ }^{16} \mathrm{O},{ }^{56} \mathrm{Fe},{ }^{235,8} \mathrm{U}$ and ${ }^{239} \mathrm{Pu}$ - with the aim of improving the accuracy of the data and resolving previous discrepancies in our understanding. This multi-laboratory pilot project, coordinated via the OECD/NEA Working Party on Evaluation Cooperation (WPEC) Subgroup 40 with support also from the IAEA, has motivated experimental and theoretical work and led to suites of new evaluated libraries that accurately reflect measured data and also perform well in integral simulations of criticality.
\end{abstract}

\section{Introduction}

The CIELO project [1], coordinated by NEA/WPEC Subgroup 40 since 2013, has stimulated advances to the neutron cross section evaluations of nuclides that significantly impact our nuclear technologies: oxygen, iron, and uranium and plutonium isotopes. The benefits

a e-mail: mbchadwick@lanl.gov of a CIELO-coordinated effort between experts in nuclear science from around the world has led to the advances described in this paper.

Computational nuclear science and computing advances have played a key role in CIELO's progress. Fast computers have enabled large-scale nuclear criticality and transport simulations, mostly with the MCNP code, to assess the performance of proposed evaluation changes,

(C) The Authors, published by EDP Sciences. This is an Open Access article distributed under the terms of the Creative Commons Attribution License 4.0 (http://creativecommons.org/licenses/by/4.0/). 
with a feedback loop leading to the optimization of the reaction model parameters and ultimately of the evaluated data files. Nuclear reaction theory and modeling codes for coupled channels, statistical reactions and fission, and R-matrix, continue to be refined. The community is also starting to understand the benefits, and use of, sensitivity tools such as the NEA's NDaST codes to help focus research efforts. Also, various insights from the NEA/WPEC Subgroup 39 adjustment project have been useful.

Experimental work has always been the foundation of nuclear reaction data evaluations, and must remain so despite the costs and time involved in executing new measurement concepts to determine cross sections to unprecedented accuracy. The rallying of efforts behind CIELO has led to measurements over the course of this pilot project, most notably measurements at IRMM/Geel, CERN, RPI, Los Alamos, and TUNL.

The CIELO project has worked with the IAEA standards project to stay abreast of standards cross section advances, and remain consistent with them. This pertains to recommendations on actinide fission and capture cross sections. A new standards evaluation will be released in 2017 [2], and will be documented in a Nuclear Data Sheets issue in January 2018.

\section{CIELO evaluations created}

The CIELO pilot project has a goal of resolving some previous discrepancies in the evaluated data, via peer review interactions together with new experiments, theory, and simulation. But it is also recognized that - in some cases - differences of opinion will persist, reflecting open unsolved problems and uncertainties. In these cases the goal is to document the differences (see Refs. [1,3-5]) and reflect them in alternate data evaluations. We account for this diversity by creating and archiving two sets of files, CIELO-1 and CIELO-2, with each set of files designed to work together as a suite in criticality applications. Many of the cross section updates have compensating impacts on criticality. For example in thermal systems involving uranium and oxygen, the increased criticality from the lower average-energy ${ }^{235} \mathrm{U}$ prompt fission neutron spectrum (PFNS) is partly compensated by the changes to ${ }^{235} \mathrm{U}$ capture (increase) and oxygen that lower the criticality (increased $(n, \alpha)$ leads to more neutron absorption; and a lower scattering cross section leads to more leakage and less moderation).

In practice, CIELO-1 is being considered for adoption in the ENDF community (for example, they are used in ENDF/B-VIII.0-beta2), and CIELO-2 in the JEFF community. These are illustrated in Table 1. Because of space limitations, this paper provides figures from just the CIELO-1 data compilation.

\section{3. ${ }^{235} \mathrm{U}$ neutron reactions}

Prior to CIELO, evaluation projects have been strongly influenced by the uranium-235 resonance analyses by Derrien and Leal, used in many of the world's various libraries. Previous higher energy neutron cross section evaluation work in the US was led by Young and Chadwick, and Madland for PFNS, (LANL); in Europe by Romain, Morillon (CEA), and Vladuca and Tudora for
Table 1. Lead laboratories evaluating CIELO1,2 databases. CIELO-1 is being adopted by ENDF, CIELO-2 by JEFF. Many other labs contributed, including with data measurements.

\begin{tabular}{lll}
\hline Isotope & CIELO-11 & CIELO-2 \\
\hline \hline${ }^{16} \mathrm{O}$ res. & LANL/IRMM & IRSN/IRMM \\
${ }^{16} \mathrm{O}$ fast & LANL & LANL \\
\hline${ }^{56} \mathrm{Fe}$ res. & IRSN & IRSN \\
${ }^{56} \mathrm{Fe}$ fast & BNL/IAEA/CIEA & JEFF \\
\hline${ }^{235} \mathrm{U}$ res. & ORNL/IRSN & IRSN/ORNL \\
${ }^{235} \mathrm{U}$ fast & IAEA+LANL PFNS & CEA \\
\hline${ }^{238} \mathrm{U}$ res. & IRMM & IRMM \\
${ }^{238} \mathrm{U}$ fast & IAEA +LANL PFNS & CEA \\
\hline${ }^{239} \mathrm{Pu}$ res. & ORNL/CEA & ORNL/CEA \\
${ }^{239} \mathrm{Pu}$ fast & LANL & CEA \\
\hline
\end{tabular}

PFNS, and in Japan by Iwamoto, Otuka, Chiba, Kawano, and Ohsawa for PFNS. The present CIELO evaluation work was done by Capote, Trkov, Leal, Pigni, Talou and Rising.

Major challenges facing the CIELO team included how to accommodate the use of a new softer thermal PFNS spectrum, and updated PFNS spectra at higher energies, as recommended by the standards collaboration and by the IAEA CRP [6]; new standards thermal constants; new accurate neutron capture measurement from Los Alamos and RPI; and a new "modern" statistical model evaluation of inelastic, (n,2n), and other reaction channels using the EMPIRE modeling code. Given the previous ${ }^{235} \mathrm{U}$ evaluations performed fairly well in many thermal, intermediate, and fast critical validation benchmarks, creating new evaluations that perform well has been a challenge (and one that we feel we are on our way to meeting).

A new resonance analysis has been developed by Leal (IRSN and ORNL), described separately in these proceedings; Leal's work modified the resolved resonances to account for the new LANL and RPI capture data, and better model the standards fission integral in the 7.8-11 eV range (the CIELO-2 file). Pigni (ORNL) and Trkov (IAEA) built on Leal's work with various modifications for the CIELO-1 evaluation, as described below.

The ${ }^{235} \mathrm{U}$ resolved resonance CIELO-1 evaluation recently released within the ENDF/B-VIII. $0 \beta 2$ nuclear data library has been developed on the basis of newly evaluated thermal neutron constants [2] as well as of new thermal Prompt Fission Neutron Spectra (PFNS) [6]. The softer thermal PFNS adopted in CIELO-1 increases criticality, especially for high-leakage benchmarks (and thus introduces a strong positive slope for keff v. Above-Thermal-Leakage-Fraction (ATLF) for HST (highly-enriched uranium solutions with thermal neutrons) benchmarks unless other changes are made). For energies below $100 \mathrm{eV}$, this work restores benchmark performance for ${ }^{235} \mathrm{U}$ solutions by combining changes to the prompt resonance $\bar{v}$ and the resonance parameters. In achieving this, the present set of resonance parameters yields cross sections still in reasonable agreement with the suite of experimental data included in the previous resonance 


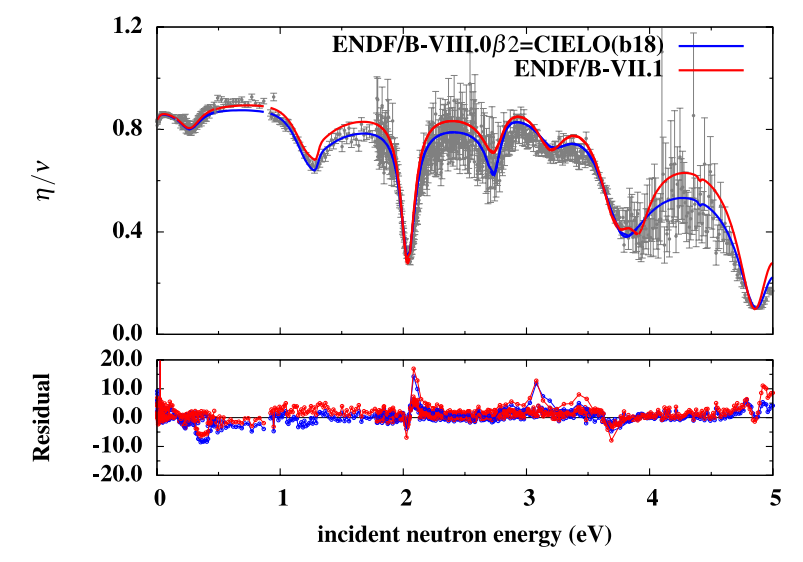

Figure 1. $\mathrm{n}+{ }^{235} \mathrm{U} \eta$ measurements of Brooks, Wartena, and Weigmann compared to ENDF/B-VII. 1 and ENDF/B-VIII.0 $\beta 2$ values.

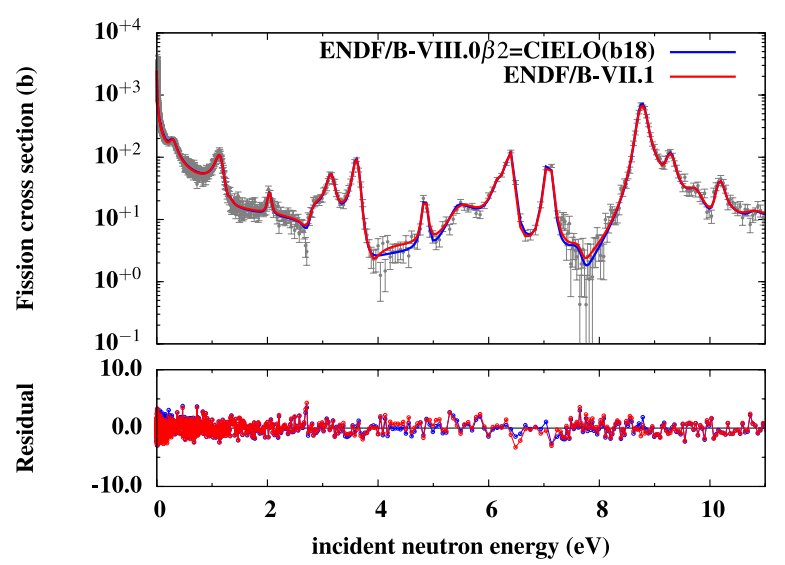

Figure 2. $\mathrm{n}+{ }^{235} \mathrm{U}$ Gwin's fission measurement compared to ENDF/B-VII. 1 and ENDF/B-VIII. $0 \beta 2$ values.

evaluations. Additionally, the set of $\eta$ measurements performed by Brooks [7] in the mid-sixties at the Atomic Energy Research Establishment (Harwell) were analyzed and included in the fit for incident neutron energies up to $20 \mathrm{eV}$. Figure 1 displays multiple measurements of Brooks [7] in the incident neutron energy range up to $5 \mathrm{eV}$ but also measured data of Wartena and Weigmann [8] in the low energy range between $0-0.5 \mathrm{eV}$. All measurements were normalized to the reported $v$ value. The comparison of ENDF/B-VII.1 (in red) and ENDF/B-VIII.0 $\beta 2$ (in blue) $\eta$ values is also shown. Despite the large uncertainties above $2 \mathrm{eV}$, the CIELO $\eta$ (decreased) values are on average in better agreement with the experimental data than ENDF/B-VII.1 values. This was achieved by increasing the capture cross sections mostly in the valley of the resonances while keeping their peak values unchanged. The resonance at $E_{\mathrm{n}}=2 \mathrm{eV}$ is clearly an example.

The sensitivity of the resonance parameters to fission cross sections seems to be more relevant than to capture cross sections at neutron energies $\geq 4 \mathrm{eV}$. This effect is evident in the fission cross sections shown in blue continuous line in the Fig. 2 where Gwin's fission cross section measurements are displayed along with ENDF/ B-VII. 1 and ENDF/B-VIII. $0 \beta 2$ values.

The decreased neutron production suggested by Brooks' data also seemed consistent with the use of a softer PFNS and the newly fitted thermal neutron constants in order to compensate the decreased criticality. Moreover,

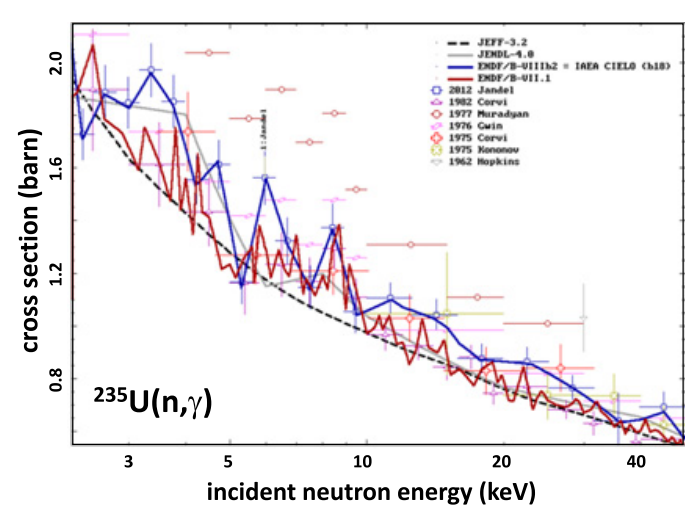

Figure 3. ${ }^{235} \mathrm{U}(\mathrm{n}, \gamma)$ neutron capture.

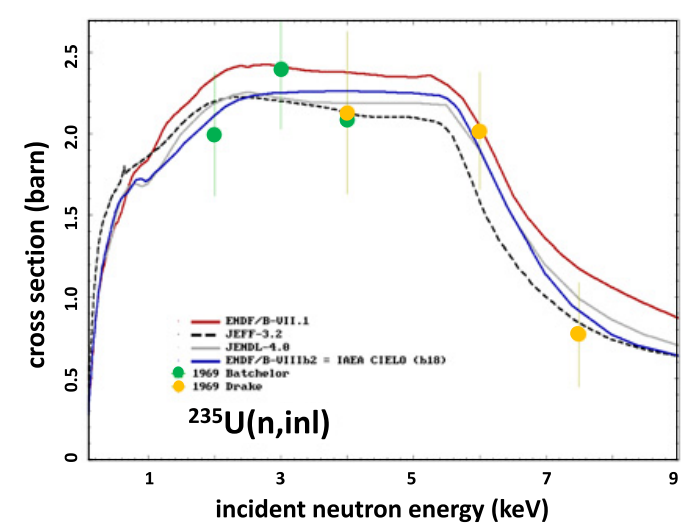

Figure 4. ${ }^{235} \mathrm{U}(\mathrm{n}, \gamma)$ total inelastic cross section.

the values of the resonance parameters were constrained by cross section integrals, e.g. the fission integral in the incident energy range between $7.8-11 \mathrm{eV}$,

$$
I_{f}=\int_{7.8 \mathrm{eV}}^{11 \mathrm{eV}} \sigma_{f}(E ; \mathbf{a}) d E=247.0 \mathrm{~b} \cdot \mathrm{eV}(\text { current value })
$$

recommended by newly nTOF measured cross section data.

The CIELO-1 ${ }^{235} \mathrm{U}$ capture cross section above one $\mathrm{keV}$ is shown in Fig. 3. It follows the recent Jandel Los Alamos measurement, lying significantly below ENDF/ B-VII.1 below $2 \mathrm{keV}$ and above it for energies up to $50 \mathrm{keV}$.

The inelastic scattering cross section has been reevaluated as part of a new optical and statistical model analysis of direct and compound reactions. CIELO's total inelastic scattering is reduced compared to ENDF/B-VII.1, see Fig. 4. At higher incident energies above $10 \mathrm{MeV}$, preequilibrium processes become important. These, together with inelastic scattering reactions involving the excitation of collective states, are included in EMPIRE model calculations, allowing for the modeling of $14 \mathrm{MeV}$ secondary neutron emission data measured by Kammerdiener at Livermore shown in Fig. 5.

The importance of the need for a better understanding of the prompt fission neutron spectra (PFNS) from actinides, owing to its large impact on criticality calculations, led to a multi-year IAEA Coordinated Research Project, the results of which are now documented in a major article [6]. An important conclusion was that the PFNS from thermal neutrons on ${ }^{235} \mathrm{U}$ should have a lower 


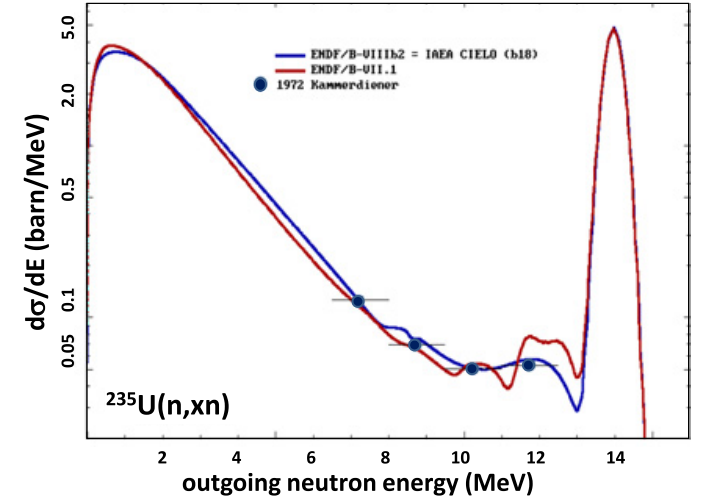

Figure 5. ${ }^{235} \mathrm{U}(\mathrm{n}, \mathrm{xn})$. CIELO's secondary neutron spectra, for $14 \mathrm{MeV}$ incident energy, compared to measurements and to ENDF/B-VII.1. Fission neutrons are included.

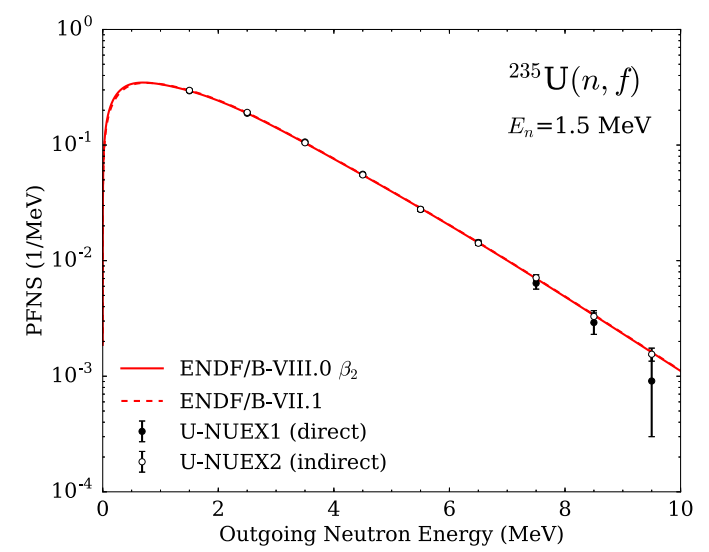

Figure 6. ${ }^{235} \mathrm{U}$ (n,PFNS). CIELO's prompt fission neutron spectra compared to NUEX data and to ENDF/B-VII.1, for $1.5 \mathrm{MeV}$ incident energy.

average energy, 2.00 MeV, versus the previous $2.03 \mathrm{MeV}$, based on an IAEA analysis of spectra and dosimetry activation measurements ${ }^{1}$. At higher neutron energies 0.5 up to $5 \mathrm{MeV}$ incident energy - CIELO adopts the calculated values by Rising and Talou which were based on an extension of the Madland-Nix model, calibrated to measured data, and applied consistently through a suite of uranium isotopes. This spectrum is seen to agree fairly well with the NUEX data of Lestone and Shores in Fig. 6 for incident neutrons with an average energy of about $1.5 \mathrm{MeV}$. It is evident from the average PFNS energies shown in Fig. 7 that the Talou-Rising PFNS data matches the new IAEA spectrum average energy at thermal, and removes the previous ENDF/B-VII.1 unphysical kink in the neutron average energy near $3 \mathrm{MeV}$ (which was based on matching one particular data set, that of Boykov). Above $5 \mathrm{MeV}$ the ENDF/B-VII.1 PFNS is maintained, although it is recognized that an upgrade is eventually needed to properly account for preequilibrium processes above $10 \mathrm{MeV}$ incident energy.

\section{4. ${ }^{238} \mathrm{U}$ neutron reactions}

Prior to CIELO, evaluation projects have been strongly influenced by the uranium-238 resonance analyses by

\footnotetext{
${ }^{1}$ This is a flashback to the past. Watt's seminal 1952 Physical Review paper parameterized the data of the time with a functional form that had an average energy of $2.00 \mathrm{MeV}$ !
}

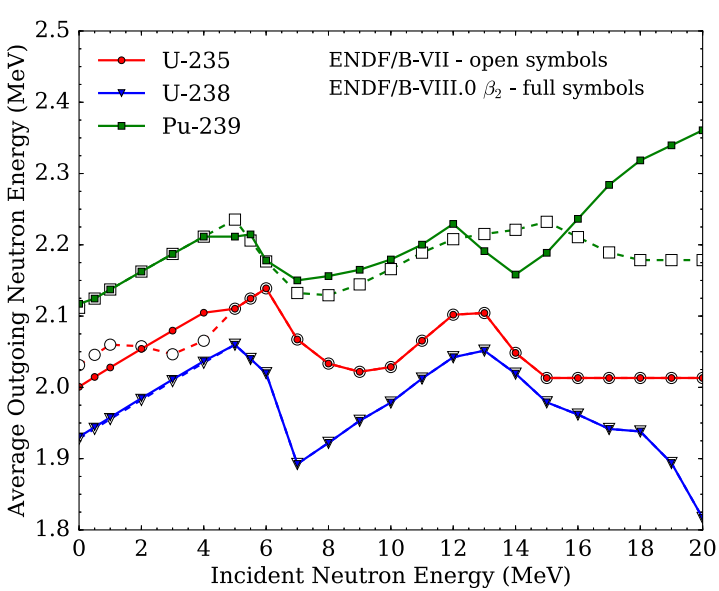

Figure 7. Major actinide averaged prompt fission neutron energy in CIELO versus ENDF/B-VII.1.

Derrien, Courcelle, Leal, and Froehner, used in many of the world's various libraries. Previous higher energy neutron cross section evaluation work in the US was led by Young and Chadwick, and Madland for PFNS (LANL); in Europe by Romain, Morillon (CEA), and Vladuca and Tudora for PFNS, and in Japan by Iwamoto, Otuka, Chiba, Kawano, and Ohsawa for PFNS.

The present CIELO evaluation work was done by Capote, Trkov, Schillebeeck, Kopecky, Talou and Rising. It involves both a new resonance analysis that takes advantage of new measurements, and a new analysis of fast reactions using a coupled-channels optical model treatment, together with Hauser-Feshbach and preequilibrium modeling of compound and direct reaction processes and fission. A full description of the CIELO evaluations on ${ }^{238} \mathrm{U}$ will be provided in forthcoming publications, and here we just provide a brief summary of the advances.

A new evaluation for neutron induced reaction on ${ }^{238} \mathrm{U}$ in the resonance region was carried out considering well documented experimental data in the literature. Resonance parameters of individual resonances below $1200 \mathrm{eV}$ were adjusted from a simultaneous resonance shape analysis of capture data obtained at GELINA [9] and transmission data obtained at a $42 \mathrm{~m}$ and $150 \mathrm{~m}$ station of ORELA $[10,11]$. The contribution of the bound states was adjusted to produce a parameter file that is fully consistent with these data. This is illustrated in Fig. 8 which compares the experimental $\mathrm{T}_{\text {exp }}$ and theoretical transmission $\mathrm{T}_{M}$ for the uranium sample with a $0.175 \mathrm{at} / \mathrm{b}$ areal density. Using the parameters of ENDF/B-VII.1, which are adopted from Derrien et al. [12], the theoretical and experimental transmission are not consistent. This suggests that Derrien et al. [12] applied a normalization correction to the experimental transmission to get a consistent fit.

In the unresolved resonance region average capture and total cross sections were derived from a least squares analysis of experimental data reported in the literature using the GMA code. The generalised ENDF-6 model together with the standard boundary conditions was used to parameterise these average cross sections in terms of average parameters following a procedure described in Ref. [13]. The neutron strength functions and hard sphere scattering radius were adjusted to reproduce results of optical model calculations using the DCCOM potential of 


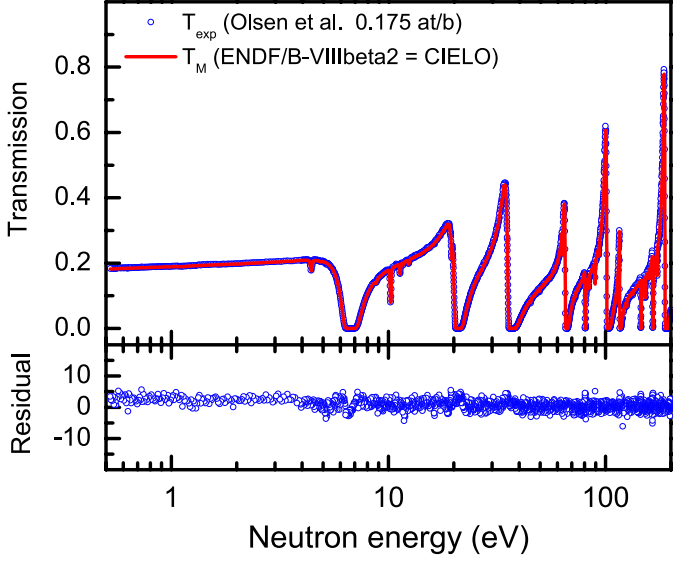

Figure 8. Resonance analysis of new $238 \mathrm{U}$ data. Schx details.

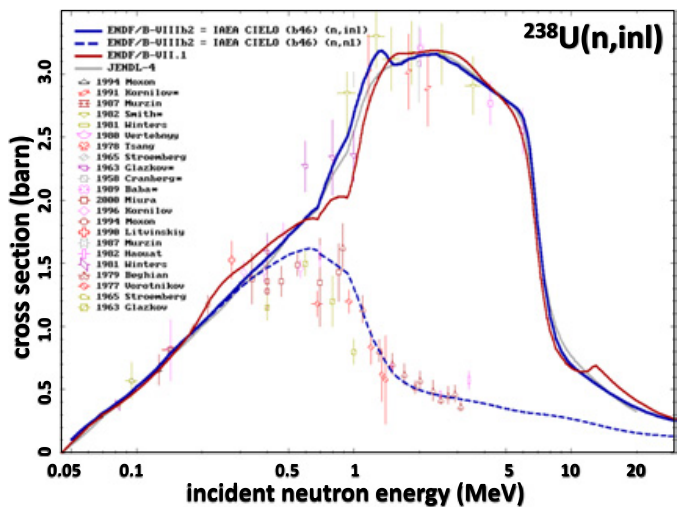

Figure 9. ${ }^{238} \mathrm{U}$ (n, inelastic).

Quesada et al. [14] and the inelastic neutron scattering data of Capote et al. [15], which include compound-direct interference effects, were adopted.

The ${ }^{238} \mathrm{U}$ inelastic scattering cross section has been a focus of attention in the CIELO collaboration, owing to its large impact on simulations of fast reactor criticality. The new evaluation shown in Fig. 9 is based on advanced nuclear reaction theory predictions, which include improved nuclear structure treatments and fission competition modeling (since accurate measurements of inelastic scattering are challenging). The role of theory is enhanced owing to the difficulty of accurately measuring scattering to the many excited states, although $(n, x \gamma)$ data can be useful to infer these reactions [16].

The evaluated ${ }^{238} U(n, 2 n)$ cross section is shown in Fig. 10, compared to ENDF/B-VII.1 and to data. The earlier ENDF/B-VII.1 evaluation rose to higher values in the $6-8 \mathrm{MeV}$ region above the threshold compared to some of the other evaluations, and this same behavior is seen in the new CIELO evaluation, informed in part by new Krishichayan measurements from TUNL. The previous VII.1 evaluation was motivated by old LANL Knight data, together with integral measurements of $(n, 2 n)$ reaction rates in critical assemblies, and this behavior is corroborated by the TUNL and other measurements, which guided the model calculations used in the present analysis.

\section{5. ${ }^{239} \mathrm{Pu}$ neutron reactions}

Prior to CIELO, evaluation projects have been strongly influenced by the plutonium resonance analyses by

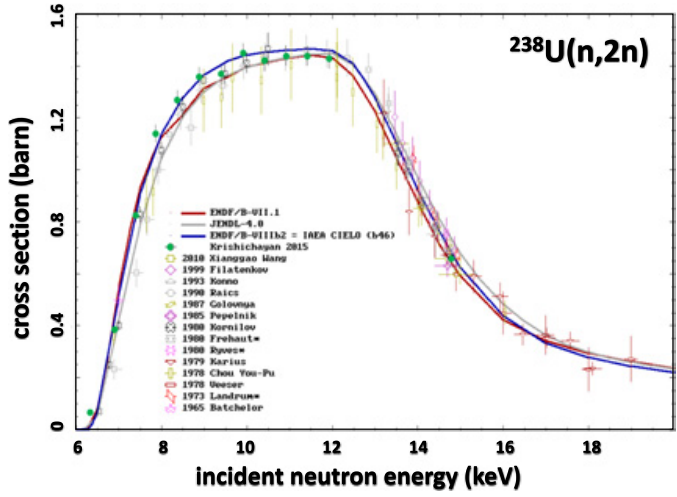

Figure 10. ${ }^{238} \mathrm{U}(\mathrm{n}, 2 \mathrm{n})$ excitation function in CIELO, compared to data that include the recent TUNL measurements.

Derrien, Leal, Larson, de Saussure, Fort, and Nakagawa. Higher energy neutron cross section plutonium evaluation work in the US was led by Young, Arthur, Chadwick, Talou, and MacFarlane, and Madland for PFNS (LANL); in Europe by Romain, Morillon, and Delaroche (CEA); and in Japan by Iwamoto, Otuka, Chiba, and Kawano.

In the last three years CIELO collaboration made only modest advances to ${ }^{239} \mathrm{Pu}$, adopting the earlier WPEC Subgroup 34 work on resonances by de Saint Jean, Noguere, Peneliau, Bernard, Serot, Leal, Derrien, Kahler, and McKnight, and by updating the ENDF capture cross sections in the fast range between $30 \mathrm{keV}$ and $2 \mathrm{MeV}$.

Earlier evaluations, such as ENDF/B-VII and JEFF-3.1, JENDL-4.0 suffered from a longstanding deficiency: an overprediction of plutonium solution criticality in transport simulations by approximately $500 \mathrm{pcm}(0.5 \%$ in $\mathrm{k}$-eff $)$ [17]. The proposed resonance and prompt nubar updates by Subgroup 34 remove approximately half of this over-prediction. The further influence of our ${ }^{16} \mathrm{O}$ CIELO evaluation changes, and the new scattering kernels recommended by WPEC/Subgroup 42, now lead to much-improved thermal plutonium solution criticality predictions as discussed below in Sect. 8 .

Additional improvements must follow this pilot project. In the coming years we expect to see new plutonium prompt fission spectra (PFNS) and fission cross section data from the Los Alamos Chi-nu and TPC experiments and use of newly evaluated thermal PFNS from the IAEA CRP [6]; as an interim step we included the Neudecker PFNS spectrum for incident energies above $5 \mathrm{MeV}$, which provides an improved treatment of the effects of multi-chance fission and preequilibrium processes. Also, the recent Mosby et al. DANCE capture data should impact a future plutonium resonance analysis in the unresolved and resolved resonance regions, analogous to how DANCE data influenced the ${ }^{235}$ U CIELO evaluation described above. In the fast region above $30 \mathrm{keV}$, these data motivated the capture cross section change shown in Fig. 11 (MBC - comment on how this change relates to the subgroup 39 adjustment capture change recommendation).

\section{6. ${ }^{56} \mathrm{Fe}$ neutron reactions}

A new effort by the CIELO collaboration to improve iron cross sections was deemed important based on 


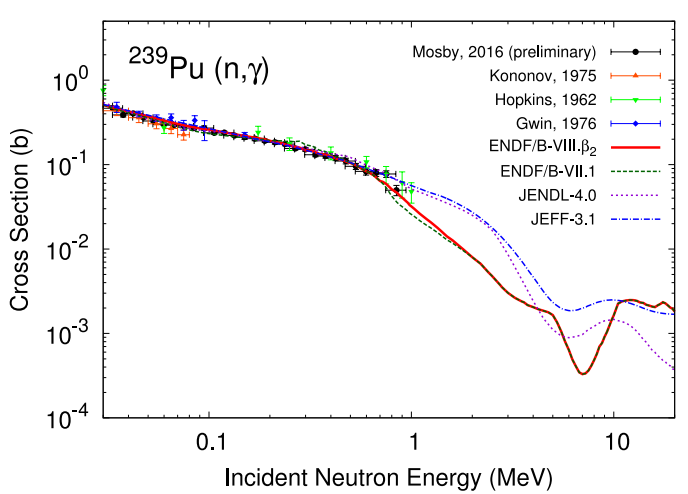

Figure 11. ${ }^{239} \mathrm{Pu}(\mathrm{n}, \gamma)$ in CIELO versus ENDF/B-VII.1, showing the influence of the new Los Alamos data.

sensitivity studies of nuclear criticality and shielding, and thermal and fast reactor design work. For example, uncertainty assessments performed by WPEC Subgroup 26 for innovative reactor systems shows that the knowledge of the inelastic scattering cross section of ${ }^{56} \mathrm{Fe}$ should be improved to meet the target accuracy requirements for these systems.

The previous ${ }^{56} \mathrm{Fe}$ evaluations in the various libraries from different regions are largely independent, with some exceptions such as the resolved resonance parameters. They rely on the optical model and statistical model calculations, where the secondary particle energy and angular distributions play an important role in radiation shielding calculations. The evaluations can be separated into four energy ranges: (a) the resolved resonance region up to $850 \mathrm{keV}$, (b) from $850 \mathrm{keV}$ to about $7 \mathrm{MeV}$ where fluctuations still persist in the measured total cross section, (c) from about $7 \mathrm{MeV}$ to $20 \mathrm{MeV}$, and (d) above $20 \mathrm{MeV}$. F. Perey and C. Perey of ORNL evaluated the resolved resonance parameters for ENDF/B-VI, and ENDF/B-VII.1 and CENDL have the same resonance parameter set. Other evaluations (JENDL, JEFF, and ROSFOND) adopt a modified version of the resolved resonances by Fröhner, performed for the JEF-2.2 evaluation.

In the $\mathrm{MeV}$ energy region, the fluctuation behavior seen in the experimental total cross sections, which an optical model cannot reproduce, should exist in the evaluated files, as this can be important in neutron transport and shielding calculations. Usually the total cross sections in this energy region are obtained by tracing the experimental data available. For the other reaction channels, the Hauser-Feshbach model calculations are used for the evaluation, though the model codes employed are different.

The CIELO evaluation is based on a new resonance analysis from Leal (IRSN) up to an energy of $850 \mathrm{keV}$, using the LRF $=7$ extension above the first inelastic level, together with guidance on angular distributions from simulations of iron-reflected critical assemblies and RPI "quasi-differential" scattering data [18]. Up to $4 \mathrm{MeV}$, the evaluated data for total, elastic, and inelastic scattering is based on measured data. At higher energies, EMPIRE nuclear model calculations played an important role, including the use of a soft-rotor optical model potential. The present CIELO work was performed by Herman, Nobre, Brown, Arcilla, Trkov, Capote, Leal, Plompen, Danon, Qian, Ge, Liu, Hanlin, Ruan, and Sin.

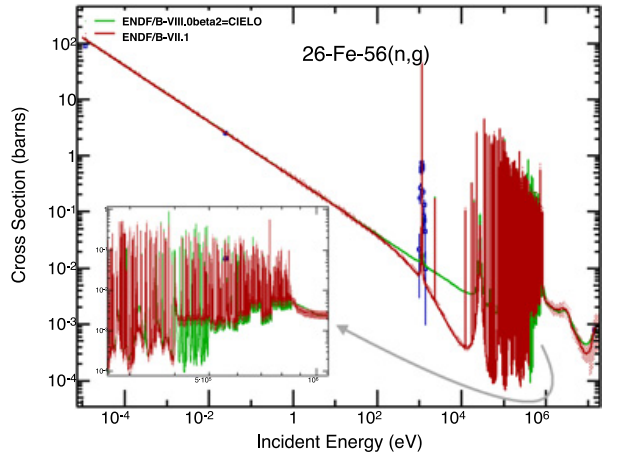

Figure 12. ${ }^{56} \mathrm{Fe}$ (n,capture) in CIELO versus ENDF/B-VII.1.

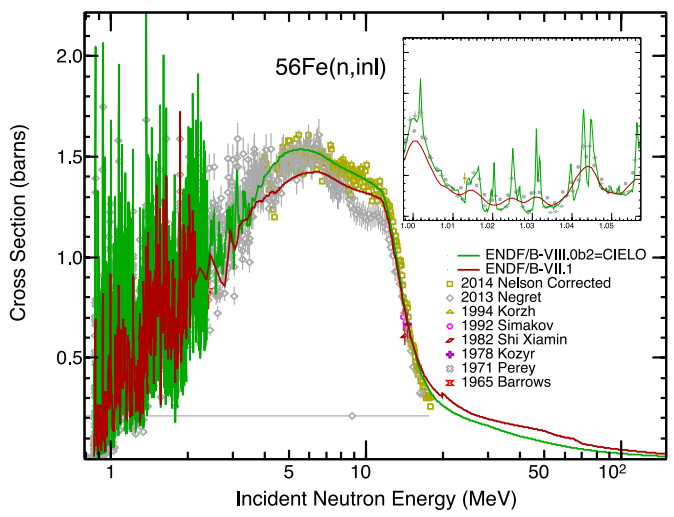

Figure 13. ${ }^{56} \mathrm{Fe}$ (n,inelastic) in CIELO versus ENDF/B-VII.1.

Leal's IRSN-v2 resolved resonance analysis was adopted up to the first inelastic scattering level $(850 \mathrm{keV})$. This analysis included the use of new RPI data [19]. As in previous evaluations, "background" modifications to the cross sections above $500 \mathrm{keV}$ were added by Trkov, to account for hypothesized missing $p$ - and $d$-wave resonances and to avoid an unphysically-low neutron capture cross section. In addition, a background was added in the $10 \mathrm{eV}-100 \mathrm{keV}$ region to extend the " $1 / \mathrm{v}$ " dependence (see Fig. 12), motivated in part by a desire to better model the ZPR-34/9 critical assembly.

The inelastic scattering in CIELO up to $4 \mathrm{MeV}$ follows experimental data from Geel (Negret) and from Dupont (unpublished; renormalized by Trkov); the latter data having a higher resolution, which is reflected in CIELO versus the earlier VII.1 evaluation. Above $4 \mathrm{MeV}$, EMPIRE model calculations are used, which were validated against the Negret and the Nelson (Los Alamos) measurements of inelastic scattering followed by gamma-ray emission. Compared to ENDF/B-VII.1, the inelastic scattering cross section in CIELO is larger, for the energy range from threshold up to $14 \mathrm{MeV}$ (Fig. 13).

\section{7. ${ }^{16} \mathrm{O}$ neutron reactions}

The existing ENDF database comes from a merging of R-matrix analyses by Hale of LANL above $3.4 \mathrm{MeV}$, and by Lubitz and Caro of KAPL below $3.4 \mathrm{MeV}$, together with higher energy data from measurements and model calculations by Young and Chadwick. This evaluation has been adopted by (or at least strongly influenced) many other evaluation projects, for example JEFF3.2, CENDL, and ROSFOND. But the CIELO researchers recognized 
that some significant modifications are now warranted; for example, a previous renormalization of the $(n, \alpha)$ cross section downwards by $32 \%$ for ENDF/B-VII is now removed, as described below. We note that this conclusion differs from that summarized in our CIELO document at the beginning of the project three years ago [1]. CIELO evaluation work for oxygen has been perfomed by Hale, Leal, Lubitz, Kunieda, Plompen, Kopecky, Kawano, (Livermore) and others. Two sets of evaluations were created for testing: Hale's (CIELO-1), and Leal's (CIEL0-2), the latter having two options for the $(\mathrm{n}, \alpha))$ cross section that can be studied.

The ${ }^{16} \mathrm{O}(\mathrm{n}, \alpha)$ reaction is important in nuclear criticality applications involving oxide fuels, and water, and its inverse - the ${ }^{13} \mathrm{C}(\alpha, \mathrm{n})$ reaction - plays an essential role in nucleosynthesis studies, being a major source of neutrons in the s-process responsible for many of the elements produced above the iron peak.

Hale, Paris and Kunieda have been making the point, for over a decade, that $\mathrm{R}$ matrix calculations constrained by unitarity, together with ${ }^{16} \mathrm{O}$ total and elastic scattering data, point to the need for a significantly higher $(n, \alpha)$ cross section in the $3-6 \mathrm{MeV}$ range. This view was adopted for the CIELO evaluation, where the cross section was increased by $\sim 40 \%$ over this range, with further increases as the incident energy extends to $9 \mathrm{MeV}$. Even though the Nuclear Energy Agency (NEA) NDaST sensitivity calculations of Hill show a very small sensitivity of the most-sensitive benchmarks to this cross section (about 3 pcm per \% change in $(n, \alpha))$, because of the very large change in the cross section one finds significant changes in calculated criticality.

The $(\mathrm{n}, \alpha)$ experimental data (and its inverse) supporting this change have been analyzed by Giorginis. Some progress has been made on clarifying what can only be said to be a messy state of affairs. The normalization of this reaction has been poorly understood in many experiments, and data have often been revised over the years. For example, the important Bair and Haas ORNL data are viewed by Giorginis as attractive owing to the use of a thin target, but to still need a renormalization close to the originally-published values (he recommends 0.95 ) instead of the larger 0.80 renormalization down that was recommended by Bair and Haas. Giorginis recommends renormalizing the Harissopulos data by 1.42 . These assessments were based on a procedure to first determine the relative scale of these two measurements based on the thick-target yield over the narrow resonance at $1.056 \mathrm{MeV}$, and subsequently to correct for issues associated with the characterization of the ${ }^{13} \mathrm{C}$ targets used in the experiments. He recommends renormalizing up his own IRMM data, first published at the Nice ND2007 conference, to be consistent with the 0.95-normalized Bair and Haas data, though so far Georginis has distributed IRMM data only in the 6.3-9 MeV range. (We assume that the Obninsk IPPE data remain in contradiction with the scale of new recommendation).

So although progress has perhaps been made, it is recognized that future experiments are needed to corroborate the large approximately $+40 \%$ changes being made in CIELO-1 (Fig. 14). Indeed, new experimental efforts have been initiated by Los Alamos (Hye Young Lee et al.) using the LENZ detector, and by astrophysical groups pursuing low-background

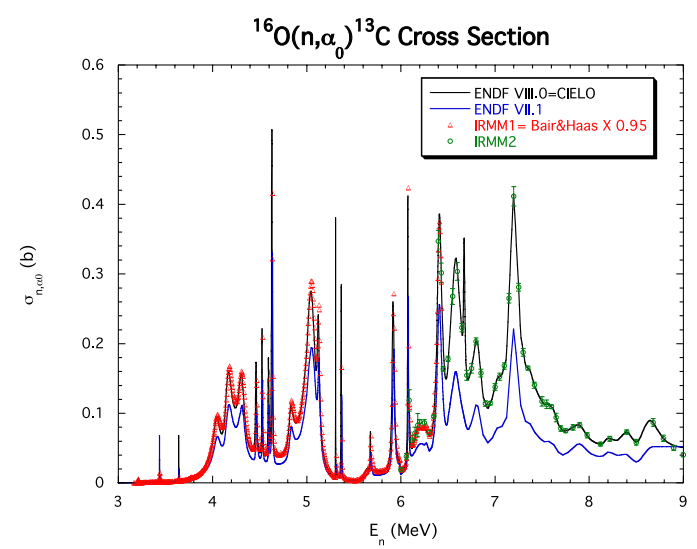

Figure 14. ${ }^{16} \mathrm{O}(\mathrm{n}, \alpha)$ in CIELO versus ENDF/B-VII.1, showing the higher cross section in the new evaluation.

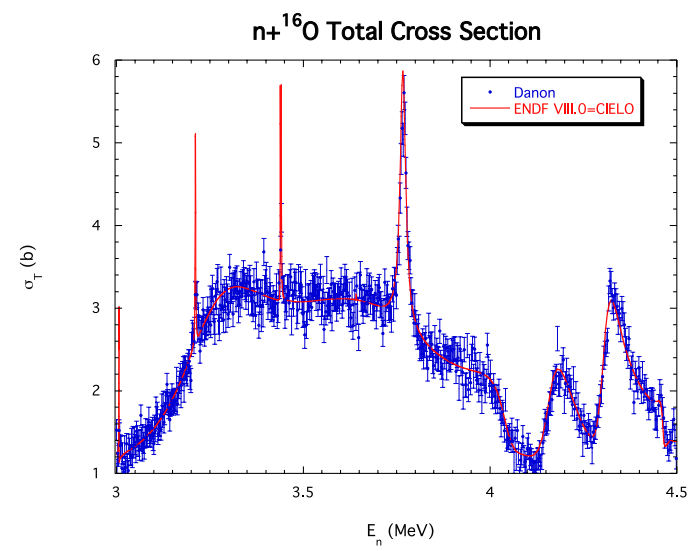

Figure 15. ${ }^{16} \mathrm{O}(\mathrm{n}, \mathrm{tot})$ total cross section in CIELO, calculated prior to the measured RPI data from Danon.

underground measurements (Wiescher et al.), and we look forward to the publication of these data.

The total ${ }^{16} \mathrm{O}$ (n,tot) cross section plays an important role in our understanding of neutron reactions in oxygen, in part because of its influence on the $(n, \alpha)$ reaction via unitarity. Historically there have been questions at the $3-4 \%$ level regarding the absolute normalization of this cross section: for example the Cierjacks 1968 data being discrepant with the high-resolution Cierjacks 1980 data. Danon et al. [20] have advanced our understanding here with a novel method in which the normalization of a measurement using a water target was made at $2.3 \mathrm{MeV}$, where the oxygen "window" (where the total cross section falls to almost zero owing to a destructve interference effect) allows the normalization to be made to the very well known hydrogen standard value. These new RPI data agree with Cierjacks 1968 to about $0.04 \%$. These measurements were also treated as blind validation data, and Fig. 15 shows they largely support the new Hale evaluation, which was done prior to the measurement. The Hale evaluation agrees with the Danon RPI total cross section data to better than $1 \%$ over the energy range from $0.2-9 \mathrm{MeV}$. It is now thought that the Cierjacks 1980 total cross section data need to be renormalized up by approximately $3.2-3.8 \%$.

The other important change for oxygen is the lower total elastic scattering cross section adopted, from thermal to $10 \mathrm{~s}$ of $\mathrm{keV}$ energies. An assessment by Kopecky and Plompen led to a recommendation of a low-energy value of 3.765 b (CIELO-2); Hale's latest value of just under 3.8 


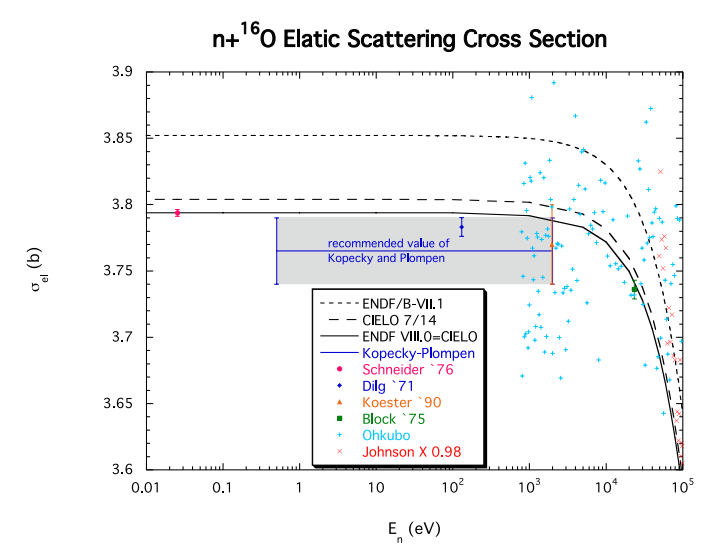

Figure 16. ${ }^{16} \mathrm{O}$ (n,elastic) total elastic scattering cross section in CIELO at low energies.

barns in the CIELO-1 file - which was influenced also by the Schneider (1976) measurement - is about $1.5 \%$ lower than the previous ENDF/B-VII.1 evaluation (3.852b), Fig. 16. This seemingly-modest decrease has a significant impact on criticality applications (for example, the NDaST sensitivity tools indicate HST benchmarks are sensitive at the $100 \mathrm{pcm}$ per $\%$ change in the elastic scattering cross section between $1 \mathrm{eV}$ and $100 \mathrm{keV}$ ). Kozier, Roubtsov, Plompen and Kopecky [21] have noted that some heavywater criticality benchmarks also suggest a lower thermal scattering cross section.

\section{Criticality validation testing}

Validation testing of CIELO files was done throughout the evaluation um void reactivity in fast FCA assemblies.

We have used the NEA's NDaST sensitivity tools to assess the impact of some of the CIELO-1 cross section changes, relative to ENDF/B-VII.1. Below we use the changes to ${ }^{16} \mathrm{O}(\mathrm{n}, \alpha)$ and (n,elastic) as an illustrative example. Hill has analyzed over 3000 criticality benchmarks to characterize the effects.

The role of the increased CIELO- ${ }^{16} \mathrm{O}(\mathrm{n}, \alpha)$ reaction in absorbing neutrons and reducing criticality was found to be of order $-100 \mathrm{pcm}$ on LCT experiments, and about $-50 \mathrm{pcm}$ for HST experiments. The reduced low energy elastic scattering in CIELO, on the other hand, was found to be about $-50 \mathrm{pcm}$ on LCTs (but a higher value, $-150-200 \mathrm{pcm}$ on heavy water benchmarks), while for HST experiments the reduction is about $-100 \mathrm{pcm}$ for low-leakage systems (owing to reduced moderation), but as high as $-300 \mathrm{pcm}$ for high-leakage systems (where reduced scattering increases the leakage). The overall effects is that simulations of HST highly-enriched solution thermal critical assemblies typically change by $-100-200 \mathrm{pcm}$, whereas LCT low-enriched uranium thermal assemblies change by $-150-200 \mathrm{pcm}$. Some heavy water benchmarks change by almost $-300 \mathrm{pcm}$. As noted earlier, compared to ENDFB-VII.1, these reductions in criticality are compensated (in part at least) by other changes to the ${ }^{235} \mathrm{U}$ resonance and nubar data and the thermal PFNS.

For plutonium solution thermal (PST) critical assemblies, previous ENDF/B-VII and earlier JEFF and JENDL libraries largely overcalculated the criticality, by $\sim 500 \mathrm{pcm}$ on average. The adoption of Subgroup 34's plutonium resonances and nubar in CIELO removed about one half of this discrepancy. The aforementioned changes to oxygen further reduced the overprediction by $100-200$ pcm with an average effect of about $150 \mathrm{pcm}$ (of which about $3 / 5$ was due to the reduced elastic channel, and $2 / 5$ to the increased $(n, \alpha))$. Further small reductions came from the adoption of the new scattering kernel for water, and from the use of a very slightly harder thermal PFNS for plutonium.

\section{Future work}

The CIELO pilot project is ending in 2017. Ongoing work on covariances for the CIELO data will be included into the files. The progress made will be documented in a WPEC Subgroup 40 summary report, and the CIELO-1, CIELO-2 data libraries will be archived at the NEA and IAEA. Additional detailed documentation will be provided in a series of papers to be published in the January 2018 issue of Nuclear Data Sheets, edited by Pavel Oblozinsky.

The CIELO covariance data need particular attention. In the ENDF community, ENDF/B-VII.1 had a focused effort on providing covariances for a large range of isotopes and reactions. Nevertheless, numerous questions remain, especially when comparing ENDF/B-VII.1 uncertainty assessments with those in JENDL and JEFF files, and even when comparing uncertainties assessed in the resonance range versus the fast range within a given library. The Nuclear Energy Agency WPEC Subgroup 39 has provided a valuable assessment of such questions and discrepancies, in a paper by Dr. Ishikawa [22]. This useful paper pointed out that - even for very important reactions such as major actinide fission, capture, and inelastic scattering differences in uncertainties as large as an order-ofmagnitude are not uncommon, for certain energy regimes. The CIELO project hopes to help resolve some of these questions.

There are additional details regarding the evaluated data for CIELO nuclides that have intentionally not been addressed in the CIELO collaboration, owing to time and scope limitations. Fission product yields were not studied, although another NEA WPEC subgroup (Subgroup 37) led by R.W. Mills has made progress on yields based on theory advances incorporated in Dr. Schmidt's GEF code and new measured data that have been reported. The IAEA is also coordinating much-needed studies on fission product data. Another topic is inelastic scattering. It has been central to the CIELO advances, but we were limited in the amount of time available for subject matter experts to work collaboratively across different laboratories to resolve differences. The challenges were laid out in a useful IAEA document by Plompen et al. [4]. But there remain open questions on magnitude of the inelastic cross sections, as well as the merits of different treatments for angular distributions in both the $\mathrm{MeV}$ and the 10sof $\mathrm{MeV}$ preequilibrium regions, ranging from quantum to semiclassical approaches [23-25].

We feel that the CIELO collaboration has stimulated much progress in nuclear experiments, theory, evaluation, and simulation. Many of the results will be adopted by regional evaluation efforts, such as ENDF and JEFF. In the long term, the community is considering the best way to continue such collaborative efforts in nuclear science, under the auspices of the IAEA and NEA. 
The LANSCE facility at Los Alamos has provided valuable data for these CIELO evaluations. The facility supports the kind of data needs described in this paper, as well measurements in basic science using ultracold neutrons, radiography, medical isotope production, electronic chip testing for single-event upsets [26], and material science using neutron scattering. In the coming five years, the precision fission experiments (Chi-nu, TPC, and SPIDER), and NEUANCE upgrades to our DANCE detector, will come to fruition and provide additional data to support these CIELO evaluations.

Work at Los Alamos National Laboratory was carried out under the auspices of the National Nuclear Security Agency of the U.S. Department of Energy under Contract No. DE-AC5206NA25396. Work at Brookhaven National Laboratory was sponsored by the Office of Nuclear Physics, Office of Science of the U.S. Department of Energy under Contract No. DE-AC0298CH10886 with Brookhaven Science Associates, LLC. Work at Lawrence Livermore National Laboratory was performed under Contract DE-AC52-07NA27344 and Oak Ridge National Laboratory under contract DE-AC05- 00OR22725. This paper is approved for release as LA-UR-16-26943 (2016).

\section{References}

[1] M.B. Chadwick, E. Dupont, E. Bauge et al., "The CIELO Collaboration: Neutron Reactions on ${ }^{1} \mathrm{H}$, ${ }^{16} \mathrm{O},{ }^{56} \mathrm{Fe},{ }^{235} \mathrm{U},{ }^{238} \mathrm{U}$ and ${ }^{239} \mathrm{Pu} "$, Nucl. Data Sheets 118, 1 (2014)

[2] V.G. Pronyaev et al, "New fit of neutron thermal constants for ${ }^{233,235} \mathrm{U},{ }^{239,241} \mathrm{Pu}$ and ${ }^{252} \mathrm{Cf}$ : microscopic vs integral data," abstract 703, ND2016 International Conference on Nuclear Data and Technology

[3] E. Bauge et al., Eur. Phys. J. A 48, 113 (2012)

[4] A. Plompen et al., International Atomic Energy Agency Report INDC(NDS)-0597 (2012)

[5] M.B. Chadwick, M.W. Herman, P. Obložinský et al., et al. "ENDF/B-VII.1 Nuclear Data for Science and Technology: Cross Sections, Covariances, Fission Product Yields and Decay Data”, Nucl. Data Sheets 112, 2887 (2011)

[6] R. Capote et al, "Prompt Fission Neutron Spectra of Actinides," Nuclear Data Sheets 131, 1 (2016)

[7] F.D. Brooks et al., "Eta and neutron cross sections of ${ }^{235} \mathrm{U}$ from 0.03 to $200 \mathrm{eV}$," AERE-M1670

[8] H. Weigmann et al.,"Measurements of Eta of ${ }^{235} \mathrm{U}$ for Subthermal Neutron Energies," Conf: Int.Conf.on the Physics of Reactors, Marseille 1990, 3, p. 33 (1990), France

[9] H.I. Kim, C. Paradela, I. Sirakov, B. Becker, R. Capote, F. Gunsing, G.N. Kim, S. Kopecky, C. Lampoudis, Y.-O. Lee, R. Massarczyk, A. Moens, M. Moxon, V.G. Pronyaev, P. Schillebeeckx and R. Wynants, Eur. Phys. J. A 52, 170 (2016)

[10] D.K. Olsen, G. de Saussure, R.B. Perez, E.G. Silver, F.C. Difilippo, R.W. Ingle, H. Weaver, Nucl. Sci Eng. 62, 479 (1977)

[11] D.K. Olsen, G. de Saussure, R.B. Perez, F.C. Difilippo,R.W. Ingle, Nucl. Sci. Eng. 66, 141 (1978)
[12] H. Derrien, L.C. Leal, N.M. Larson, A. Courcelle, Neutron Resonance Parameters and Calculated Cross Sections from Reich-Moore Analysis of Experimental Data in the Neutron Energy Range from 0 to $20 \mathrm{keV}$, ORNL/TM-2005/241, Oak Ridge National Laboratory (2005)

[13] I. Sirakov, R. Capote, F. Gunsing, P. Schillebeeckx, A.Trkov, Ann. Nucl. Energy 35, 1223 (2008)

[14] J.M. Quesada, R. Capote, E. Sh. Soukhovitskii, S. Chiba, Nucl. Data Sheets 118, 270 (2014)

[15] R. Capote, A. Trkov, M. Sin, M. Herman, A. Daskalakis,Y. Danon, Nucl. Data Sheets 118, 26 (2014)

[16] N. Fotiades et al., "Measurements and calculations of U238(n,xn $\gamma$ ) partial gamma-ray cross sections", Phys. Rev. C 69(2), 024601 (2004)

[17] A. Kahler et al., "ENDF/B-VII.1 Neutron Cross Section Data Testing with Critical Assembly Benchmarks and Reactor Experiments", Nucl. Data Sheets 112, 2997 (2011)

[18] Y. Danon et al., "Recent Developments in Nuclear Data Measurementsat the Gaerttner LINAC Center at RPI", Proc. of WONDER 2015: Fourth International Workshop on Nuclear Data Evaluation for Reactor Applications, Aix-en-Provence, France, October 5-8, 2015

[19] B. McDermott et al., "Iron-56 capture cross section experiments at the RPI LINAC center", ND2016 International Conference on Nuclear Data for Science and Technology, Bruges, Belgium, 11-16 September 2016

[20] Y. Danon et al., "Measurement of total cross section of water and O-16 in the MeV range", 12th. International Topical Meeting on Nuclear Applications of Accelerators (AccApp'15), Washington DC, November 2015

[21] K. Kozier, D. Roubtsov, A Plompen, S. Kopecky, Proc. PHYSOR 2012 - Advances in Reactor Physics, April 15-20 (2012)

[22] M. Ishikawa, "Comments on Covariance Data of JENDL-4.0 and ENDF/B-VII.1", April 22, 2014, Minutes of Joint SG39/SG40CIELO meeting, May 14, 2014. See https : //www . oecd-nea.org/science/wpec/ sg40-cielo/Meetings/2014_May/Adjustment/

[23] T. Kawano, R. Capote, S. Hilaire, and P. Chau Huu-Tai,"Statistical Hauser-Feshbach theory with width-fluctuation correction including direct reaction channels for neutron-induced reactions at lowenergies", Phys. Rev. C 94, 014612 (2016)

[24] M. Dupuis et al. "Progress in microscopic direct reaction modeling of nucleon induced reactions", Eur. Physical Journal A 51, 168 (2015)

[25] M.B. Chadwick and P. Oblozinsky "Particle-hole state densities with linear momentum and angular momentum in preequilibrium reactions", Phys. Rev. C 46(5), 2028 (1992)

[26] M.B. Chadwick and E. Normand, "Use of new ENDF/B-VI proton and neutron cross sections for single event upset calculations", IEEE Transactions on Nuclear Science 46(6), 1386 (1999) 